\title{
A nomogram with lymph node status predicts survival and the benefit of postoperative adjuvant therapy in IIIA-N2 Non-small cell lung cancer patients after surgery
}

\section{Ye Chen}

Zhejiang Chinese Medical University

\section{Lei Dong}

Zhejiang Chinese Medical University

Minjing Li

Zhejiang Chinese Medical University

Fei He

Zhejiang Chinese Medical University

\section{ChenHui Qiu}

Zhejiang Chinese Medical University

Wanru Cai ( $\nabla$ xhcaiwanru@163.com)

The Second Affiliated hospital of Zhejiang Chinese Medical University https://orcid.org/0000-00023073-2566

\section{Research}

Keywords: Non-small cell lung cancer, prognosis, nomogram, postoperative radiotherapy

Posted Date: December 7th, 2020

DOI: https://doi.org/10.21203/rs.3.rs-98065/v2

License: (a) (i) This work is licensed under a Creative Commons Attribution 4.0 International License. Read Full License 


\section{Abstract}

Objective: This study aimed at establishing a novel nomogram predicting overall survival and investigating the survival benefit of various postoperative adjuvant treatments (POAT) in IIIA-N2 Nonsmall cell lung cancer (NSCLC) patients after surgery.

Methods: Data of IIIA-N2 NSCLC patients between 2004 and 2016 were collected from the Surveillance, Epidemiology, and End Results (SEER). Patients were excluded if the information regarding follow-up time and clinicopathological features were incomplete. Through Univariate and multivariate analyses, independent prognostic factors were identified and integrated into the construction of nomogram. The survival benefit of POAT was evaluated in model-defined low-risk, intermediate-risk, and high-risk subgroups, respectively.

Results: In total, 4389 patients were finally included for analysis. Patients' age, sex, T stage, differentiation grade, examined lymph nodes number (ELN), metastatic lymph nodes number (MLN), and metastatic lymph nodes ratio (LNR) were identified as independent prognostic factors and were integrated into the construction of nomogram. The $\mathrm{C}$-index and calibration curves indicated that the predictive performance of the nomogram was satisfactory. Patients were then categorized into three prognostic groups with the increasing risk of all-cause of death. The prognosis of patients receiving POAT (POCT or PORT plus POCT) and patients receiving surgery alone was comparable in low-risk group, while POCT could significantly prolong survival for IIIA-N2 NSCLC patients after surgery in moderate-risk and high-risk groups. Only patients in high-risk group could benefit from the combination of postoperative radiotherapy (PORT) and postoperative chemotherapy (POCT).

Conclusion: In this large-cohort retrospective study, A survival-predicting nomogram and risk stratification model were established to estimate prognosis in IIIA-N2 NSCLC patients. Surgery alone was recommended as the first choice of treatment to patients in low-risk group. POCT was recommended for patients in moderate-risk group, and the combination of PORT and POCT was recommended for patients in high-risk group. This study may provide additional integration, introspection, and improvement for therapeutic decision-making.

\section{Introduction}

Lung cancer (LC) is the second most commonly diagnosed cancer in the United States and the leading cause of death in cancer both in women and men. There are an estimated more than 20,000 morbidity and 14,000 mortality due to lung cancer in $2019^{[1]}$. Non-small cell lung cancer (NSCLC) is the most common histopathological subtype of LC, accounting for about $85 \%$ of all LC, of which squamous cell carcinoma and adenocarcinoma are the most common subtypes ${ }^{[2,3]}$. Although improvements have been made in diagnostic and therapeutic techniques, many cases were still diagnosed at an advanced stage with poor diagnosis ${ }^{[4]}$. 
Curative resection for primary lung cancer and metastatic lymph nodes is still the first choice for the majority of NSCLC patients. Nevertheless, for locally advanced NSCLC, especially N2 diseases, the optimal treatment after surgery remains controversial. The existing clinical guidelines hold distinct views on the application of postoperative treatment in IIIA-N2 NSCLC patients. NCCN guidelines recommend PORT for stage N2 NSCLC patients. While American Society of Clinical Oncology and American Society for Radiation Oncology guidelines suggest that PORT is considered unable to improve survival outcomes and is not routinely recommended for patients with IIIA-N2 NSCLC $[5,6]$.

In the real world, NSCLC is a heterogeneous tumor with diverse prognostic outcomes and can be affected by many demographic factors, including age, sex, marital status, and race, as well as clinicopathological features (such as lymph node status, grade, tumor size, and clinical treatment) ${ }^{[7,8]}$. Additionally, not all patients with IIIA-N2 NSCLC could benefit from POAT. Thus, it is vital to integrate all potential information to accurately estimate the prognosis of each patient and select patients who could benefit from postoperative adjuvant therapy.

Recently, nomogram has been considered as a commonly convenient tool for assessing prognostic outcomes, especially in cancer patients. Several nomograms have been constructed to predict the risk of recurrence, metastatic risk, and radiotherapy response in lung cancer ${ }^{[9-11]}$. However, large population-based survival-predicting models for IIIA-N2 NSCLC patients have not been found. Herein, in this present study, we reported the first large population-based investigation into factors associated with the survival outcomes of patients suffering from IIIA-N2 NSCLC. Further, we established a survivalpredicting model to assist the decision-making of postoperative adjuvant treatment.

\section{Methods}

\section{Data source and case selection}

This was a retrospective study base on the data collected from the Surveillance, Epidemiology, and End Results (SEER) database, an authoritative information source of the National Cancer Institute ( $\mathrm{NCl}$ ), which covered nearly 34.6 percent of the U.S. population ${ }^{[12]}$. In this present study, information for patients with IIIA-N2 NSCLC was collected based on the following exclusion and inclusion criteria.

Exclusion criteria: (1) autopsy/death certificate diagnosed only; (2) unknown/obscure follow-up time; (3) not first tumor; (4) No/Unknown surgery; (5) distant metastasis; (6) unknown/no information on the extracted data; (7) overall survival $\leq 3$ months.

Inclusion criteria: (1) histologically confirmed diagnosis of IIIA-N2 non-small cell lung cancer; (2) active follow-up.

The time range of our study was determined based on the data availability of the sixth/ seventh AJCC TNM staging version. The collected information included basic demographics (age, race, gender), histopathologic characteristics of tumor (primary tumor location, differentiation grade, TNM 
stage(6th/7th)), details of treatment (record of surgery, chemotherapy and radiotherapy), and survival outcomes (follow-up time, and OS status). In this study, the endpoint for NSCLC patients was overall survival (OS), which was determined as the period from the date of histologic diagnosis to the date of death due to any cause or the last follow-up time.

\section{Statistical analysis}

The optimal cut-off values for examined lymph nodes number (ELN), metastatic lymph nodes number (MLN), and metastatic lymph nodes ratio (LNR) were determined using the X-tile software. Univariate and multivariate Cox proportional hazard analyses were used to identify independent prognostic variables through a backward stepwise method. Further, based on these variables, we finally built the survival-predicting nomogram with the SURVIVAL and RMS package in R software. The C-index was utilized to evaluate the discrimination ability and clinical utility of the model. We also generated the calibration curves to assess predicting accuracy of this model by comparing the actual OS with the model-predicting OS probability. Furthermore, based on the total scores of each patient, a risk stratification system was developed to divide all cases into three subgroups with different prognosis. the Kaplan-Meier method and Log-rank test were applied to compare the OS outcome between subgroups.

All statistical analyses were processed using R software (version 3.5.1; http://www.r-project.org) and SPSS 22.0 (IBM Corporation, Armonk, NY, USA). The risk stratification system was established using the X-tile software (Version 3.6.1.1, Yale University, New Haven, CT, USA). Two-side P $<0.05$ was considered as statistical significance.

\section{Results}

\section{Patient characteristics}

In total, 4389 IIIA-N2 NSCLC patients receiving curative surgery were selected from the SEER database. Among all patients, the majority (93.3\%) were older than 50 years of age. Most cases presented with pathological grade II $(\mathrm{N}=1924)$ and III $(\mathrm{N}=2085)$ at the time of diagnosis. In addition, $33.0 \%(\mathrm{~N}=1449)$ of patients had $\mathrm{T} 1$ stage and $67.0 \%(\mathrm{~N}=2940)$ had T2 stage. The median follow-up time was 40 months. The actuarial 1-year, 2-year, 3-year and 5-year OS were 85.3\%, 73.3\%, 54.0\% and $39.1 \%$, respectively. Detailed patients' clinicopathological characteristics were listed in Table 1. 
Table 1. Demographic and clinicopathological characteristics of IIIA-N2 NSCLC patients after surgery

\begin{tabular}{|c|c|c|c|c|c|c|c|}
\hline & \multicolumn{2}{|c|}{ All patients } & \multicolumn{2}{|c|}{$\overline{\text { PORT }}$} & \multicolumn{2}{|c|}{ No PORT } & \multirow[b]{2}{*}{$P$ value } \\
\hline & $\mathrm{n}=4389$ & $\%$ & $\mathrm{n}=1767$ & $\%$ & $\mathrm{n}=2622$ & $\%$ & \\
\hline$\overline{\text { Age }}$ & & & & & & & 0.000 \\
\hline$<65$ & 1915 & 43.6 & 885 & 50.1 & 1030 & 39.3 & \\
\hline$\geq 65$ & 2474 & 56.4 & 882 & 49.9 & 1592 & 60.7 & \\
\hline Gender & & & & & & & 0.344 \\
\hline Female & 2093 & 47.7 & 858 & 48.6 & 1235 & 47.1 & \\
\hline Male & 2296 & 52.3 & 909 & 51.4 & 1387 & 52.9 & \\
\hline Race & & & & & & & 0.289 \\
\hline White & 3580 & 81.6 & 1435 & 81.2 & 2145 & 81.8 & \\
\hline Black & 425 & 9.7 & 164 & 9.3 & 261 & 10.0 & \\
\hline Other & 384 & 8.7 & 168 & 9.5 & 216 & 8.2 & \\
\hline Grade & & & & & & & 0.086 \\
\hline Well & 246 & 5.6 & 82 & 4.6 & 164 & 6.3 & \\
\hline Moderately & 1924 & 43.8 & 764 & 43.2 & 1160 & 44.2 & \\
\hline Poorly & 2085 & 47.5 & 865 & 49.0 & 1220 & 46.5 & \\
\hline Undifferentiated & 134 & 3.1 & 56 & 3.2 & 78 & 3.0 & \\
\hline Laterality & & & & & & & 0.739 \\
\hline Left & 1998 & 45.5 & 799 & 45.2 & 1199 & 45.7 & \\
\hline Right & 2391 & 54.5 & 968 & 54.8 & 1423 & 54.3 & \\
\hline $\mathrm{T}$ classification & & & & & & & 0.528 \\
\hline T1 & 1449 & 33.0 & 593 & 33.6 & 856 & 32.6 & \\
\hline
\end{tabular}


T2

$2940 \quad 67.0$

$1174 \quad 66.4$

$1766 \quad 67.4$

ELN

0.008

Median(range)

$9 \quad 1-90$

$9 \quad 1-82$

9

$1-90$

MLN

0.000

Median(range)

$2 \quad 1-61$

$2 \quad 1-61$

2

$1-41$

LNR

0.000

Median(range)

0.28

0-1

0.33

0-1

0.24

0-1

\section{Independent OS-related prognostic factors}

The optimal cutoff points of ELN, MLN and LNR were determined with the highest specificity and sensitivity using the X-tile software. For ELN, its optimal cutoff values were 6 (Figure 1A); For MLN, its optimal cutoff values were 3 and 6 (Figure 1B). For LNR, its optimal cutoff value was $0,0.25$, and 0.45 (Figure 1C). The log-rank tests confirmed that the survival differences between cohorts classified by ELN, MLN or LNR were statistically significant.

Table 2 showed the hazard ratios for overall survival according to all factors (including lymph node status) in the univariate and multivariate Cox proportional hazards model. Variables, including age, sex, $T$ stage, differentiation grade, ELN, MLN, and LNR were significantly associated with prognosis in univariate analysis (Table 2, left panel), and were then enrolled into the multivariate analysis to confirm the association. Finally, age $(p<0.0001)$, sex $(p<0.0001)$, T stage $(p<0.0001)$, differentiation grade $(p<$ $0.0001), \operatorname{ELN}(p<0.0001), \operatorname{MLN}(p<0.0001)$, and LNR $(p<0.0001)$ were identified as the independent overall survival-related prognostic factors (Table 2, right panel). 
Table 2.Univariate and multivariate analysis for all-cause of death in IIIA-N2 NSCLC patients after surgery

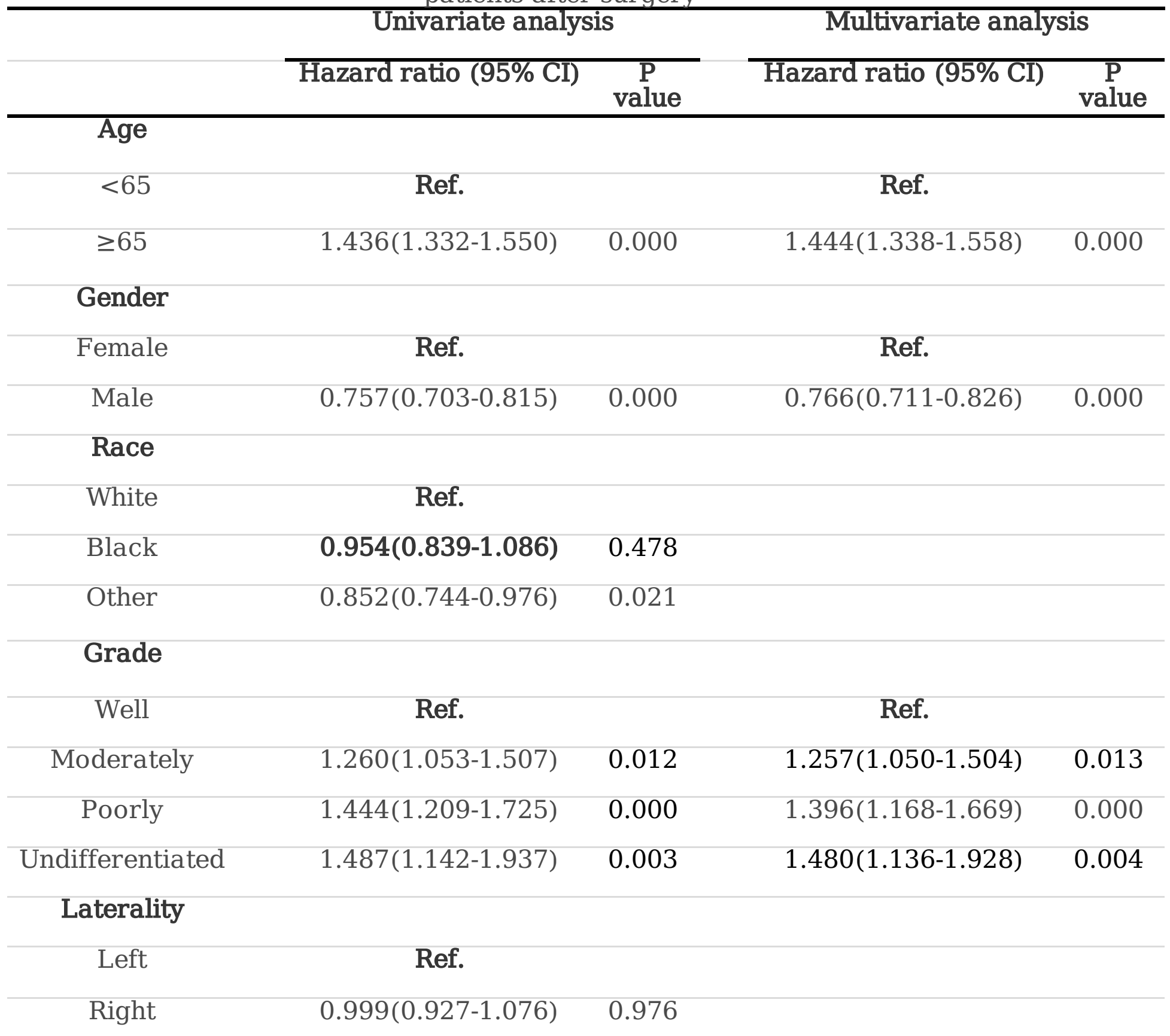

\section{T classification}

$\begin{array}{ccccc}\text { T1 } & \text { Ref. } & & \text { Ref. } & \\ \text { T2 } & 1.325(1.222-1.436) & 0.000 & 1.296(1.195-1.406) & 0.000 \\ \text { ELN } & & & & \\ <6 & \text { Ref. } & & \text { Ref. } & \\ \geq 6 & 0.840(0.771-0.914) & 0.000 & 0.831(0.742-0.930) & 0.001 \\ \text { MLN } & & & & \\ <3 & \text { Ref. } & & \text { Ref. }\end{array}$




$\begin{array}{ccccc}{[3,6)} & 1.175(1.078-1.279) & 0.000 & 1.085(0.980-1.202) & 0.115 \\ \geq 6 & 1.619(1.468-1.785) & 0.000 & 1.343(1.168-1.545) & 0.000\end{array}$

\section{LNR}
0
Ref.
Ref.
$(0,0.25]$
1.258(1.026-1.543)
0.027
1.301(1.059-1.598)
0.012
$(0.25,0.45]$
1.588(1.291-1.953)
0.000
1.501(1.209-1.863)
0.000
$>0.45$
2.213(1.797-2.724)
0.000
1.971(1.571-2.472)
0.000

\section{The generation of nomogram and risk stratification system}

According to the multivariate Cox proportional hazards model, age, sex, T stage, differentiation grade, ELN, MLN, and LNR were finally identified and integrated into the construction of a predictive nomogram for 1-, 3- and 5-year survival. Each element of these variables included was allotted a score, for example, LNR $>0.45$ had a score of 100 . The total point was calculated by adding all scores on the basis of the clinicopathologic characteristics of each patient. Finally, the likelihood of all-cause of death could be read by drawing a plummet line (Figure 2A).

The C-index values of the OS-predicting nomogram were 0.71 (95\% Cl: 0.699-0.721). The calibration curves showed that the nomogram-predicted survival outcomes had satisfying consistency with the actual prognosis results (Figure 2B).

Furthermore, based on the total scores of each patient, a novel risk stratification system was proposed to divide all IIIA-N2 NSCLC patients into three prognostic subgroups (Figure 2C), including lowrisk group (total score < 160, $N=1327$ ), intermediate-risk group (total score: [160, 215], $N=1618$ ) and high-risk group (total score $>215, \mathrm{~N}=1444$ ). Among all the cases, the median OS time was 68 months in low-risk group, 40 months in intermediate-risk group, and 26 months in high-risk group. The Kaplan-Meier analysis and the log-rank test indicated that this risk stratification system could accurately distinguish survival outcomes in the three prognostic subgroups (Figure 2C).

\section{Clinical benefit of various postoperative treatments}

First, we assessed the survival benefits of several postoperative therapy strategies (i.e. surgery alone, postoperative radiotherapy (PORT), postoperative chemotherapy (POCT), and PORT plus POCT) in all patients. The results showed that for the entire, patients receiving POCT or PORT plus POCT had better survival outcomes than patients receiving surgery alone or PORT ( $<0.001$, Figure $3 \mathrm{~A})$. Similarly, the Kaplan-Meier curves and log-rank tests were then generated in the three prognostic subgroups respectively. In low-risk group, the prognosis of patients receiving POAT (POCT or PORT plus POCT) and patients receiving surgery alone was comparable, while in moderate-risk and high-risk groups, POCT 
could significantly prolong survival for IIIA-N2 NSCLC patients after surgery. Additionally, in moderate-risk subgroup, the difference was not statistically significant in the OS of IIIA-N2 NSCLC patients who received POCT in view of whether underwent PORT (Figure 3B,3C). For patients in high-risk subgroup, the combination of PORT and POCT showed a significant advantage in prolonging OS in IIIA-N2 NSCLC patients compared with receiving POCT alone (Figure 3D).

\section{Discussion}

Accurate prediction of survival prognosis is critical for better management of NSCLC patients, and to date, this mainly relied on the AJCC TNM staging system. However, the predictive performance of the existing systems for NSCLC remains unsatisfactory ${ }^{[13]}$. In fact, even in patients with the same TNM stage, the survival outcomes remain heterogenous ${ }^{[14]}$. In this study, for the first time, a novel nomogram with lymph node status was established and validated for predicting overall survival outcomes in IIIA-N2 NSCLC patients, which is user-friendly, accurate, and practical.

Various studies had reported several variables associated with the prognosis of patients with lung cancer. $\mathrm{Li}$ et al found that $\mathrm{T}$ stage, $\mathrm{N}$ stage, the number of examined lymph nodes and age were statistically prognostic factors for surgical resected carcinoma of the lung ${ }^{[15]}$. In the present study, the prognostic values of the lymph node status in IIIA-N2 NSCLC patients receiving curative surgery were investigated. The X-tile software was utilized to explore the optimal cutoff points of ELN, MLN, and LNR. The combination of ELN, MLN, and MLNR could effectively reflect the involvement of regional metastasis and the extent of surgery, and the result of univariate analysis confirmed that these variables were prognostic predictors for IIIA-N2 NSCLC patients.

Notably, only variables of lymph node status are not sufficient to accurately reflect the postoperative prognosis of IIIA-N2 NSCLC patients. In the present study, all variables of interest were included into the multivariate analysis, and seven clinicopathologic features were finally identified as independent prognostic factors, including age, sex, T stage, pathologic grade, ELN, MLN, and LNR. Among all these variables, LNR $>0.45$ made the greatest contribution to poorer prognosis followed by undifferentiated grade and $M L N>6$. C-index and calibration curves indicated that the predictive performance of the nomogram was satisfactory.

The goal of systemic therapy in NSCLC patients is to mitigate symptom burden from NSCLC and improve survival outcomes, with a co-occurrent aim of improving the quality of life ${ }^{[16]}$. Whether adjuvant therapy is suitable for NSCLC patients was kept controversial for decades, especially since the results reported by PORT Meta-analysis Trialists Group in $1998^{[17]}$. Some previous studies reported that PORT could not prolong the survival rate of NSCLC patients compared with surgery alone ${ }^{[18]}$. In the meantime, some studies revealed that with the progress of radiotherapy technology, PORT could significantly improve the prognosis of IIIA-N2 NSCLC patients, resulting in reduced local-regional recurrence and better $0 S^{[19]}$. Notably, even in IIIA-N2 NSCLC patients, the survival outcomes are heterogeneous, and not all IIIA-N2 
NSCLC patients could benefit from the treatment of POAT. The role of POAT in prolonging overall survival should be further investigated in NSCLC patients, especially in those with IIIA-N2 NSCLC.

Personal clinicopathologic features should be considered carefully when making a therapeutic decision for each patient. And it is critical to integrate these factors to identify IIIA-N2 NSCLC patients who can benefit from various postoperative treatments. Based on the survival-predicting model, we proposed a novel risk stratification system and the survival benefits of various postoperative treatments were analyzed in each risk subgroup. The results showed that surgery alone was recommended as the first choice of treatment to patients in low-risk group. POCT was recommended for patients with IIIA-N2 NSCLC in moderate-risk and high-risk groups. Additionally, only patients in high-risk group, instead of those in low-risk and moderate-risk groups, could benefit prognostically benefit from the combination of PORT and POCT, which provided more valuable information for therapeutic decision-making.

To the best of our knowledge, this is the first comprehensive attempt to develop a populationbased nomogram that predicts the prognosis of IIIA-N2 NSCLC patients after surgery. Our survivalpredicting nomogram can be practically applied to the clinical works to predict the prognosis of IIIA-N2 NSCLC patients and facilitate clinical consultation, which is able to help to make therapeutic plans ahead on follow-up and surveillance. Furthermore, we proposed a novel patient classification strategy, which could assess the survival benefits of various postoperative treatments for each patient.

There are still several limitations in the present study which should be highlighted, including its retrospective nature and potential selection biases ${ }^{[20]}$. Furthermore, information about some potential prognostic factors, such as the detailed chemotherapy protocol, molecular or genomic data, were not available in this database. Accordingly, we failed to incorporate these parameters into our model. Future studies are warranted to improve this model by incorporating more valuable prognostic features. In addition, the majority of patients enrolled in this study were Caucasian and black, so this nomogram is expected to externally validated, especially in the Asian population. Last, the recent development of immune checkpoint inhibitors ( $\mathrm{ICls}$ ) has profoundly changed the treatment strategy of patients with IIIAN2 NSCLC, but our study failed to reveal the survival benefits of ICls on these patients. Nevertheless, we agree with the viewpoint of the SEER database that despite the limitations in the population-based analysis, "big data" will definitely continue to be an indispensable part of medical studies for the purpose of hypothesis-generating exploratory analyses ${ }^{[21]}$.

In conclusion, a novel survival-predicting nomogram and risk stratification system were established for predicting individual OS in IIIA-N2 NSCLC. This study may help physicians in making therapeutic decisions and contribute to the design of future prospective researches.

\section{Abbreviations}

NSCLC: Non-small cell lung cancer; SEER: The Surveillance, Epidemiology, and End Results; POAT: postoperative adjuvant treatments; PORT: postoperative radiotherapy; POCT: postoperative 
chemotherapy; ELN: examined lymph nodes number; MLN: metastatic lymph nodes number; LNR: metastatic lymph nodes ratio; OS: overall survival

\section{Declarations}

\section{Acknowledgements}

This study was supported by the National Natural Science Foundation of China (81503521), and Traditional Chinese Medical Science and Technology Plan of Zhejiang Province (2020ZB105)

\section{Authors' contributions}

Conception, design, and acquisition of data: Y.C., W.C., C.Q., L.D., M.L., F.H. Data collection, analysis, and interpretation: Y.C., W.C., L.D. Manuscript draft and revision: All authors. All authors read and approved the final manuscript.

\section{Funding}

the National Natural Science Foundation of China (81503521), and Traditional Chinese Medical Science and Technology Plan of Zhejiang Province (2020ZB105)

\section{Availability of data and materials}

The authors declare that all datasets generated and analyzed are available in the SEER database (https://seer.cancer.gov/).

\section{Ethics approval and consent to participate}

As this database is open-source and informed consent of patients is not re- quired, ethics committee approval by the institutional review board was not needed for this study.

\section{Consent for publication}

Not applicable.

\section{Competing interests}

The authors declare that they have no competing interests.

\section{References}

[1] Siegel RL, Miller KD, Jemal A. Cancer statistics, 2019. CA Cancer J Clin. 2019;69(1):7-34.

[2] Misra P, Singh S. Role of cytokines in combinatorial immunotherapeutics of non-small cell lung cancer through systems perspective. Cancer Med. 2019;8(5):1976-95. 
[3] Wang S, Mao W, Wang Y, Shi X, Wang W, Dai L, Zhang W. Surgical Compliance and Survival Outcomes for Patients with Stage T1-2 Non-Small-Cell Lung Cancer. Cancer Manag Res. 2020;12:3597610.

[4] Liu R, Chen Z, Yi X, Huang F, Hu G, Liu D, Li X, Zhou H, Liu Z. 9za plays cytotoxic and proapoptotic roles and induces cytoprotective autophagy through the PDK1/Akt/mTOR axis in non-small-cell lung cancer. J Cell Physiol. 2019;234(11):20728-41.

[5] Yuan C, Tao X, Zheng D, Pan Y, Ye T, Hu H, Xiang J, Zhang Y, Chen H, Sun Y. The lymph node status and histologic subtypes influenced the effect of postoperative radiotherapy on patients with $\mathrm{N} 2$ positive IIIA non-small cell lung cancer. J Surg Oncol. 2019;119(3):379-87.

[6] Rodrigues G, Choy H, Bradley J, et al (2015). Adjuvant radiation therapy in locally advanced nonsmall cell lung cancer: Executive summary of an American Society for Radiation Oncology (ASTRO) evidence-based clinical practice guideline. Pract Radiat Oncol 5:149-55.

[7] Rodrigues G, Choy H, Bradley J, Rosenzweig KE, Bogart J, Curran WJ Jr, Gore E, Langer C, Louie AV, Lutz S, Machtay M, Puri V, Werner-Wasik M, Videtic GMM. Adjuvant radiation therapy in locally advanced non-small cell lung cancer: Executive summary of an American Society for Radiation Oncology (ASTRO) evidence-based clinical practice guideline. Pract Radiat Oncol. 2015;5(3):149-55.

[8] Chansky K, Sculier JP, Crowley JJ, Giroux D, Van Meerbeeck J, Goldstraw P; International Staging Committee and Participating Institutions. The International Association for the Study of Lung Cancer Staging Project: prognostic factors and pathologic TNM stage in surgically managed non-small cell lung cancer. J Thorac Oncol. 2009;4(7):792-801.

[9] Fan L, Cao Q, Ding X, Gao D, Yang Q, Li B. Radiotranscriptomics signature-based predictive nomograms for radiotherapy response in patients with nonsmall cell lung cancer: Combination and association of CT features and serum miRNAs levels. Cancer Med. 2020;9(14):5065-74.

[10] Ma X, Cheng J, Zhao P, Li L, Tao K, Chen H. DNA methylation profiling to predict recurrence risk in stage I lung adenocarcinoma: Development and validation of a nomogram to clinical management. J Cell Mol Med. 2020;24(13):7576-89.

[11] Wang J, Chu Y, Li J, Zeng F, Wu M, Wang T, Sun L, Chen Q, Wang P, Zhang X, Zeng F. Development of a prediction model with serum tumor markers to assess tumor metastasis in lung cancer. Cancer Med. 2020;9(15):5436-45.

[12] Overview of the SEER Program. Available online: https://seer.cancer.gov/about/ overview.html. (Accessed on 30 Aug 2019).

[13] Wu LL, Liu X, Jiang WM, Huang W, Lin P, Long H, Zhang LJ, Ma GW. Stratification of Patients With Stage IB NSCLC Based on the 8th Edition of the American Joint Committee on Cancer (AJCC) Staging 
[14] Wang X, Wang Z, Pan J, Lu ZY, Xu D, Zhang HJ, Wang SH, Huang DY, Chen XF. Patterns of Extrathoracic Metastases in Different Histological Types of Lung Cancer. Front Oncol. 2020;10:715.

[15] Li H, Wang Z, Yang F, Wang J. Development and validation of a nomogram for predicting cancerspecific survival of surgical resected stage I-II adenosquamous carcinoma of the lung. J Surg Oncol. 2020;121(6):1027-35.

[16] Arbour KC, Riely GJ. Systemic Therapy for Locally Advanced and Metastatic Non-Small Cell Lung Cancer: A Review. JAMA. 2019;322(8):764-74.

[17] Postoperative radiotherapy in non-small-cell lung cancer: systematic review and meta-analysis of individual patient data from nine randomised controlled trials. PORT Meta-analysis Trialists Group. Lancet. 1998;352:257-63.

[18] Pang Z, Yang Y, Ding N, Huang C, Zhang T, Ni Y, Du J, Liu Q. Optimal managements of stage IIIA (N2) non-small cell lung cancer patients: a population-based survival analysis. J Thorac Dis. 2017;9(10):4046-56.

[19] Lally BE, Zelterman D, Colasanto JM, Haffty BG, Detterbeck FC, Wilson LD. Postoperative radiotherapy for stage II or III non-small-cell lung cancer using the surveillance, epidemiology, and end results database. J Clin Oncol. 2006;24(19):2998-3006.

[20] Zhu X, Zhao M, Zhou L, Zhang M, Cao P, Tao L. Significance of examined lymph nodes number and metastatic lymph nodes ratio in overall survival and adjuvant treatment decision in resected laryngeal carcinoma. Cancer Med. 2020;9(9):3006-14.

[21] Sun M, Trinh QD. A Surveillance, Epidemiology and End Results (SEER) database malfunction: perceptions, pitfalls and verities. BJU Int. 2016 Apr;117(4):551-2.

\section{Figures}

(A)

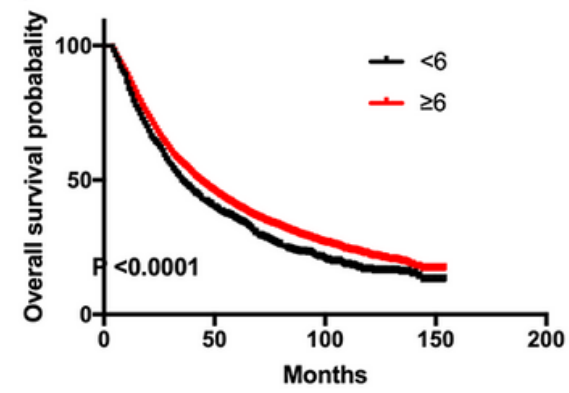

(B)

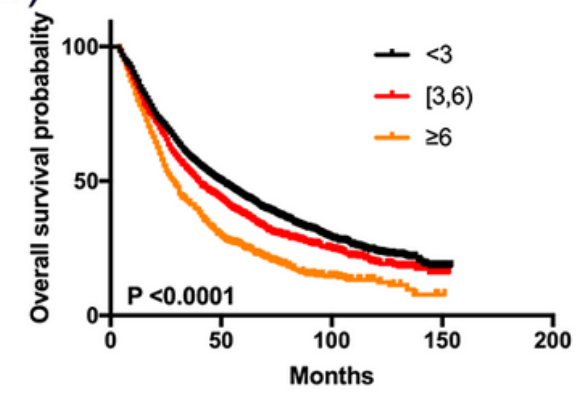

(C)

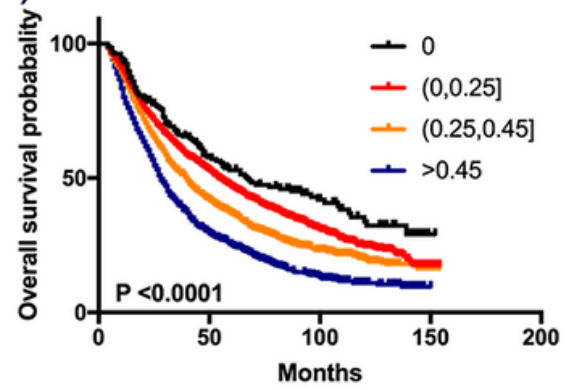


Figure 1

Optimal cut-off values determined by X-tile software for ELN (A), MLN (B), and LNR (C).

(A)

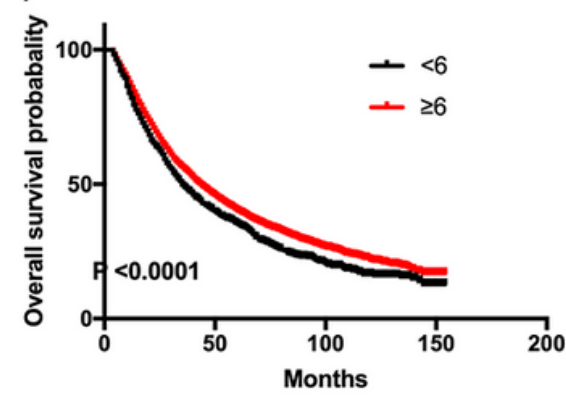

(B)

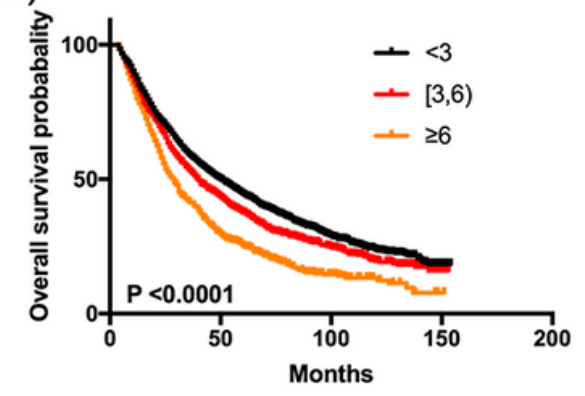

(C)

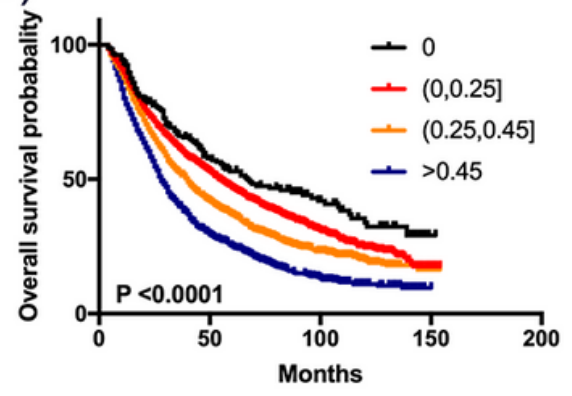

Figure 1

Optimal cut-off values determined by X-tile software for ELN (A), MLN (B), and LNR (C).

(A)

Points
Age
Sex
Grade
T.stage
ELN
MLN
LNR
Total Points
1-year OS
3-year OS
5-year OS
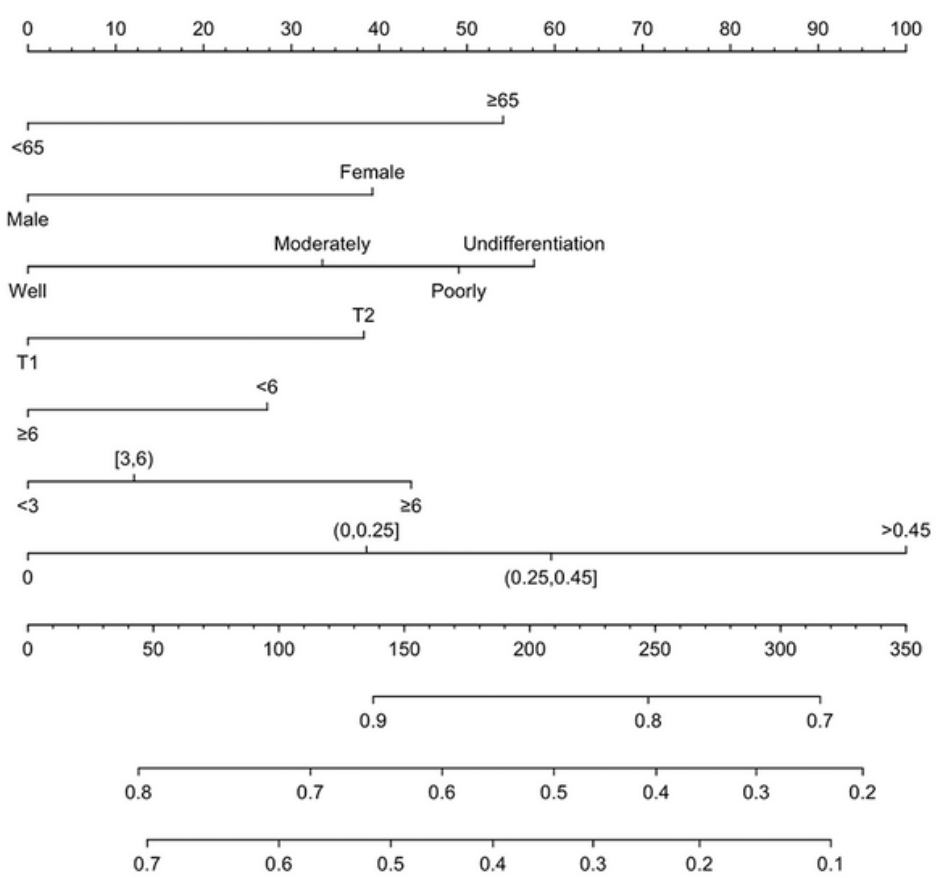

(B)

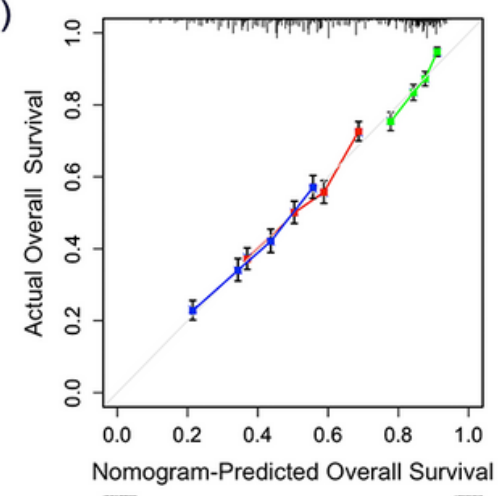

(C)

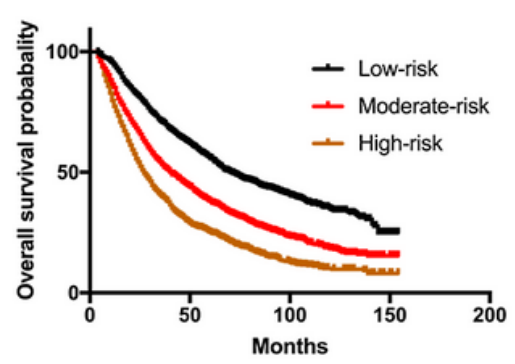

Figure 2

Nomogram for predicting 1-, 3- and 5-year overall survival in IIIA-N2 NSCLC patients after surgery (A); Calibration curve for predicting 1-, 3- and 5-year overall survival (B); Optimal cut-off values to classified patients into three prognostic subgroups by the X-tile software, including low-risk: $160<$; intermediate-risk: $[160,215) \otimes$ High-risk: $\geq 215$ (C). 
(A)

Points

Age

Sex

Grade

T.stage

ELN

MLN

LNR

Total Points

1-year OS

3-year OS

5-year OS

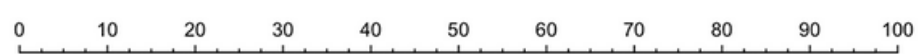
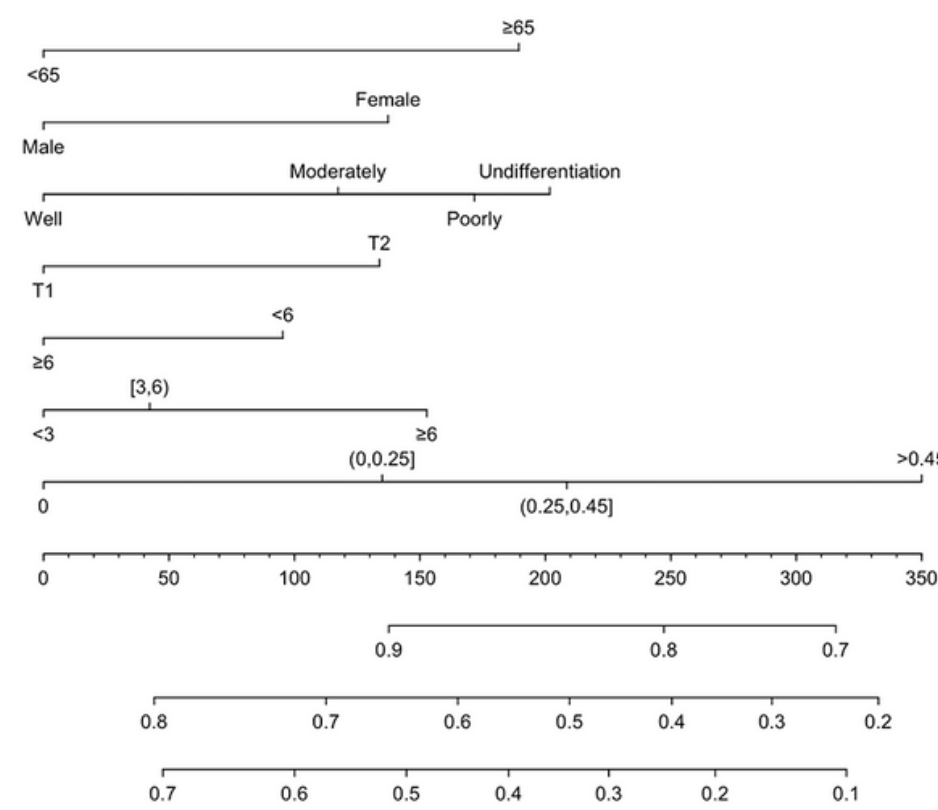

(B)

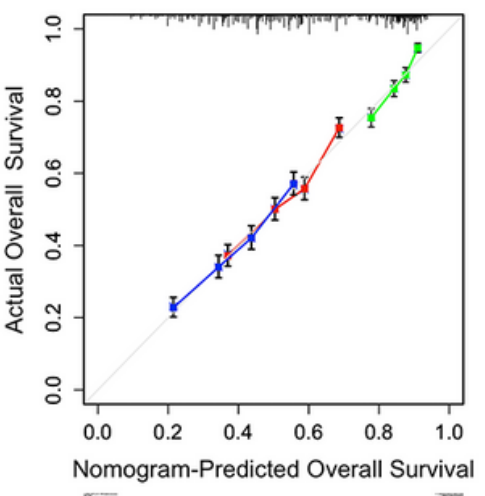

(C)

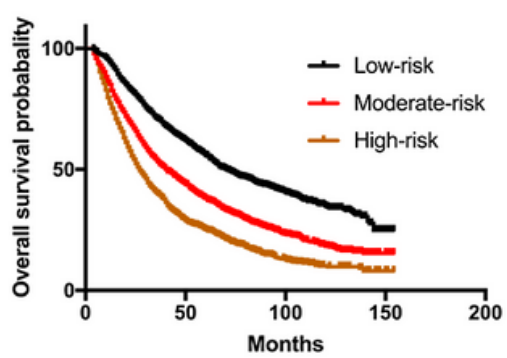

Figure 2

Nomogram for predicting 1-, 3- and 5-year overall survival in IIIA-N2 NSCLC patients after surgery (A); Calibration curve for predicting 1-, 3- and 5-year overall survival (B); Optimal cut-off values to classified patients into three prognostic subgroups by the $\mathrm{X}$-tile software, including low-risk: $160<$; intermediate-risk: $[160,215) \otimes$ High-risk: $\geq 215$ (C). 
(A)

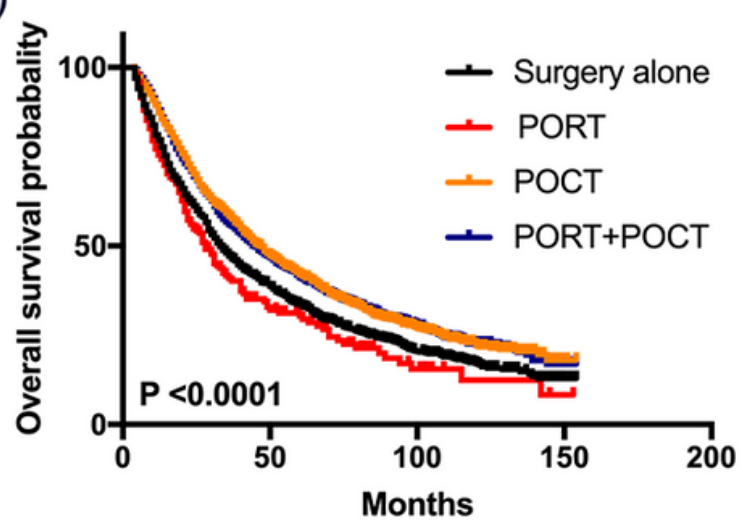

(C)

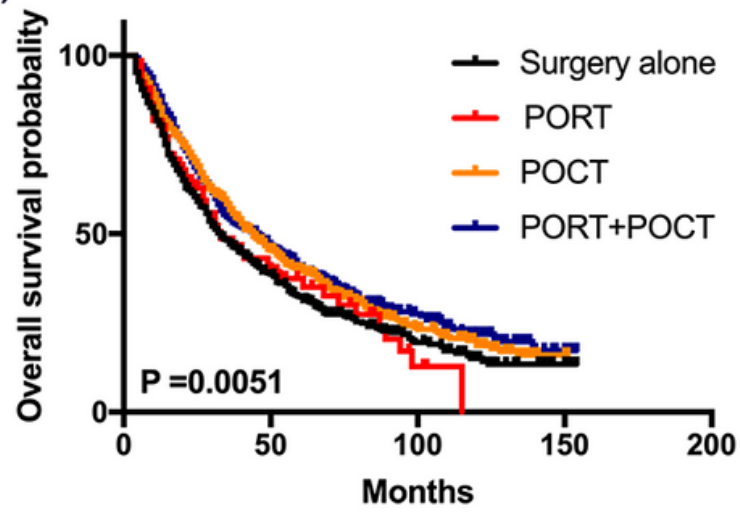

(B)

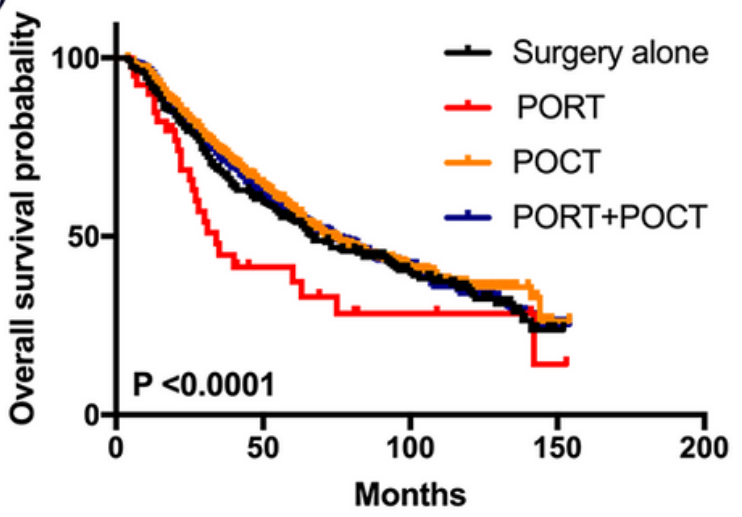

(D)

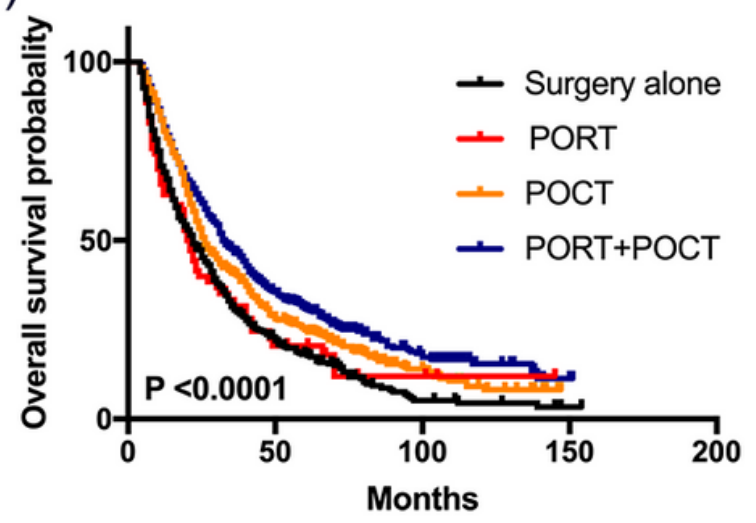

Figure 3

The survival outcomes of four therapy strategies (i.e. surgery alone, PORT, POCT, and PORT plus POCT) in all patients (A), low-risk group (B), moderate-risk group (C), and high-risk group (D). 
(A)

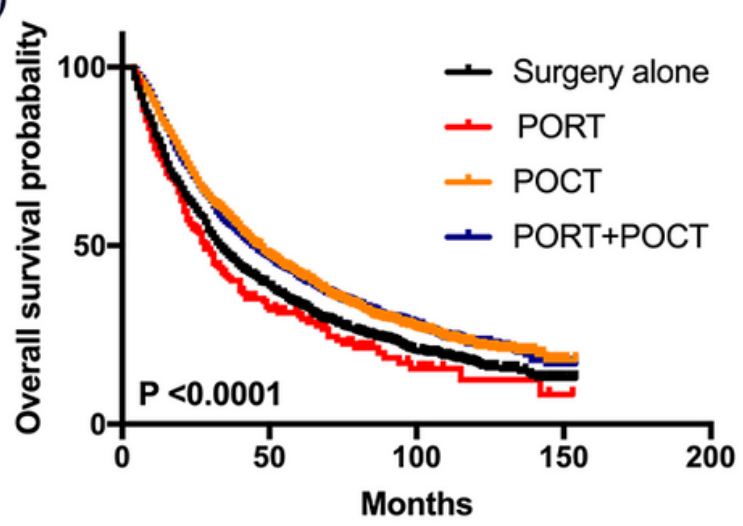

(C)

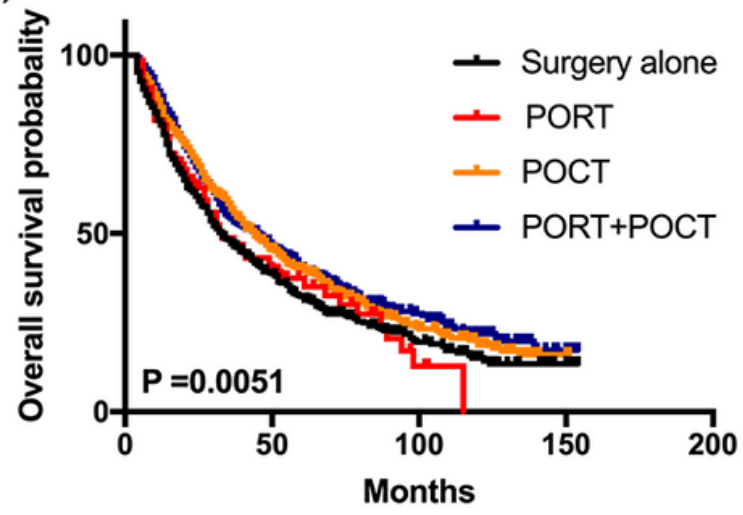

(B)

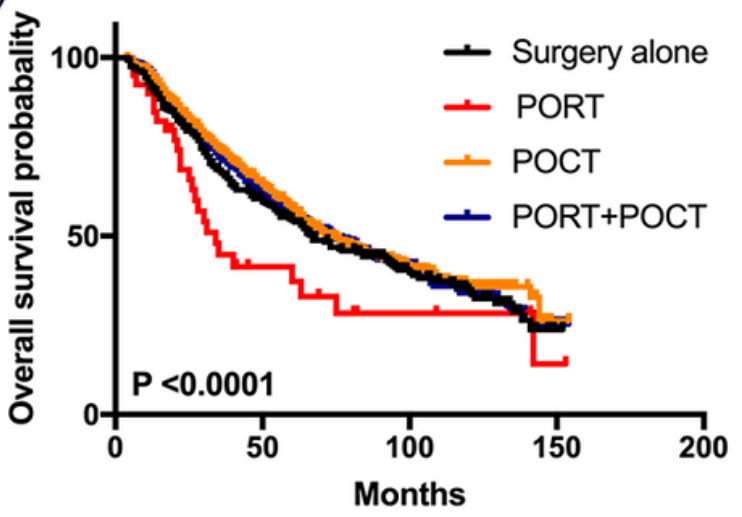

(D)

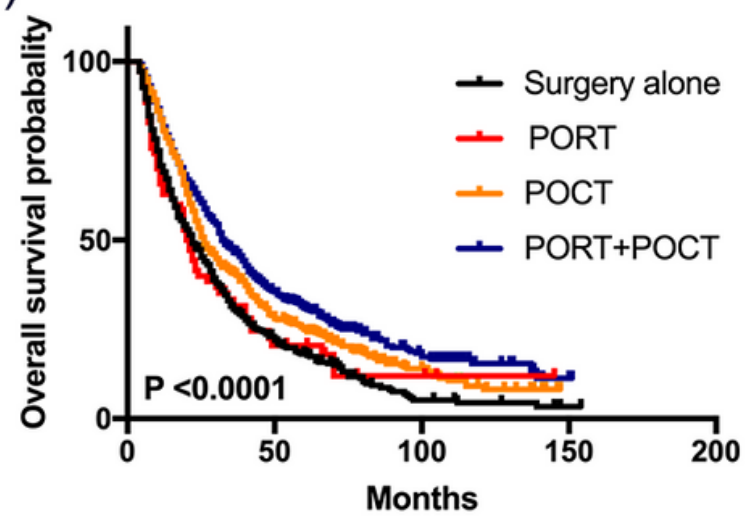

Figure 3

The survival outcomes of four therapy strategies (i.e. surgery alone, PORT, POCT, and PORT plus POCT) in all patients (A), low-risk group (B), moderate-risk group (C), and high-risk group (D). 